\title{
Diagnosis and Treatment of Implant-Associated Septic Arthritis and Osteomyelitis
}

\author{
Andrej Trampuz, MD, and Werner Zimmerli, $M D$
}

\author{
Corresponding author \\ Werner Zimmerli, MD \\ Medical University Clinic, Kantonsspital, CH-4410 Liestal, \\ Switzerland. \\ E-mail: werner.zimmerli@unibas.ch \\ Current Infectious Disease Reports 2008, 10:394-403 \\ Current Medicine Group LLC ISSN 1523-3847 \\ Copyright $(\underset{C}{2008}$ by Current Medicine Group LLC
}

Implant-associated infections are caused by microorganisms growing in biofilms, rendering these infections difficult to diagnose and to eradicate. Delayed-onset low-grade infection is difficult to distinguish from aseptic failure, often presenting without signs of infection, but only with early loosening and persisting pain. A combination of criteria is needed for an accurate diagnosis: clinical signs and symptoms, laboratory signs of infection, microbiology, histology, and imaging. The treatment goals in prosthetic joint-associated infection are eradication of infection and an optimal functional result. The goal in internal fixation device-associated infection is consolidation of the fracture and avoidance of chronic osteomyelitis. Successful treatment requires an adequate surgical procedure combined with long-term antimicrobial therapy, ideally with an agent acting on adhering biofilm microorganisms. This article reviews the epidemiology, pathogenesis, diagnosis, and management of implant-associated infections, and presents pathogenesis and risk of hematogenous infection.

\section{Introduction}

Perioperative antimicrobial prophylaxis, advanced device design, improved surgical technique, and laminar airflow operating room environments have substantially decreased the rate of implant-associated infections. Nevertheless, with increasing numbers of implanted devices, the absolute number of implant-associated infections is steadily increasing [1]. In patients with primary joint replacement, the infection rate during the first 2 years is currently less than $1 \%$ in hip and shoulder prostheses, less than $2 \%$ in knee prostheses, and less than $9 \%$ in elbow prostheses [2]. About $5 \%$ to $10 \%$ of inserted internal fixation devices become infected; the incidence of infection after internal fixation of closed fractures is generally lower $(0.5 \%-2 \%)$, whereas the incidence may exceed $30 \%$ after fixation of grade 3 open fractures. Because the follow-up in most published series is limited to a few years, and many cases of presumed aseptic failure may be due to infection, the actual incidence of device-associated infection is likely higher.

In addition, infection rates after revision surgery are usually considerably higher than after primary replacement. Prosthetic joints remain susceptible to hematogenous seeding during their entire lifetime and some perioperative infections may have a latency period longer than 2 years. Therefore, for accurate comparisons, the frequency of infection should be reported as incidence rate (per prosthesis-years) rather than as risk (without specified denominator). The incidence of infection per 1000 prosthesis-years involving hip and knee prostheses was 5.9 (95\% CI: 5.3-6.5) during the first 2 years after implantation and 2.3 (95\% CI: 2.1-2.5) during the following 8 years [3].

\section{Pathogenesis}

Implant-associated infections are caused by microorganisms growing in biofilms, which live attached to a surface in a highly hydrated extracellular matrix. Within biofilms, microorganisms develop into organized and complex communities with structural and functional heterogeneity resembling multicellular organisms. Release of cell-to-cell signaling molecules (quorum sensing) allows microorganisms in a biofilm to respond in concert by changing their gene expression involved in biofilm differentiation. Depletion of metabolic substances causes microbes to enter into a stationary state, rendering them up to 1000 times more resistant to most antimicrobial agents than their planktonic (free-living) counterparts [4].

Implant-associated infections occur either by direct inoculation into the surgical wound during surgery or immediately thereafter (perioperative infection); by microbial spread through blood from a distant focus of 


\begin{tabular}{|c|c|c|c|c|}
\hline Classification & $\begin{array}{l}\text { Prosthetic } \\
\text { joint infections }\end{array}$ & $\begin{array}{l}\text { Infections } \\
\text { associated } \\
\text { with fracture } \\
\text { fixation devices }\end{array}$ & Pathogenesis & Typical microorganisms \\
\hline Early infection & $<3 \mathrm{mo}$ & $<2 w k$ & $\begin{array}{l}\text { During implant surgery or } \\
\text { the following } 2-4 \text { days }\end{array}$ & $\begin{array}{l}\text { Highly virulent organisms such as } \\
\text { Staphylococcus aureus or gram- } \\
\text { negative bacilli }\end{array}$ \\
\hline Delayed infection & $3-24 \mathrm{mo}$ & 2-10 wk & $\begin{array}{l}\text { During implant surgery with } \\
\text { delayed manifestation }\end{array}$ & $\begin{array}{l}\text { Less virulent organisms such as } \\
\text { coagulase-negative staphylococci } \\
\text { or Propionibacterium acnes }\end{array}$ \\
\hline Late infection & $>24 \mathrm{mo}$ & $>10 \mathrm{wk}$ & $\begin{array}{l}\text { Predominantly caused by } \\
\text { hematogenous seeding } \\
\text { from remote infections }\end{array}$ & $\begin{array}{l}\text { Typically caused by virulent } \\
\text { microorganisms such as } \\
\text { S. aureus, streptococci, or } \\
\text { gram-negative bacilli }\end{array}$ \\
\hline
\end{tabular}

infection (hematogenous infection); by direct spreading from an adjacent infectious focus; or due to penetrating trauma (contiguous infection). Table 1 shows the classification of implant-associated infections according to the onset of symptoms after implantation and the most common causative organisms.

\section{Definition of Infection}

Staphylococci are the most frequent infective agents, followed by streptococci, enterococci, gram-negative bacilli, and Propionibacterium acnes [2,3]. In prosthetic joint infections, early infection is defined as appearance of the first signs and symptoms of infection at the implant site less than 3 months after surgery. This type of infection mainly occurs by the exogenous route, and is generally caused by highly virulent microorganisms (eg, Staphylococcus aureus). Delayed (low-grade) infection is defined as appearance of the first manifestation of infection 3 to 24 months after surgery. In most such cases, microorganisms of low virulence (eg, coagulase-negative staphylococci, $P$. acnes) are inoculated during surgery. Late infection is defined as the appearance of the first signs and symptoms of infection more than 24 months after surgery; most of these infections result from hematogenous seeding from a distant focus.

Infections associated with internal fixation devices are classified as early ( $<2$ weeks), delayed (2-10 weeks), and late (> 10 weeks) [5]. These infections generally occur exogenously, either by the penetrating trauma itself, during insertion of the fixation device, or during disturbed wound healing. Hematogenous infection is less frequent and is mainly caused by bacteremia originating from skin, respiratory, and urinary tract infection. Early infections are mainly caused by highly virulent organisms such as $S$. aureus or gram-negative bacilli, whereas delayed and late infections are mainly caused by microorganisms of low virulence (eg, coagulase-negative staphylococci).

\section{Diagnostic Workup}

Laboratory parameters

The blood leukocyte count and differential are not sufficiently discriminative to predict the presence or absence of infection. In Europe, the erythrocyte sedimentation rate is no longer used routinely as a diagnostic tool to detect infection. Serum C-reactive protein (CRP) is elevated after surgery, and returns to normal within weeks; therefore, repetitive measurements are more informative than a single postoperative value. An increase of C-reactive protein after an initial postoperative decline is highly suggestive of infection. The role of procalcitonin was mainly investigated in patients with respiratory tract infection, but its value in prosthetic joint infection has not yet been defined.

Synovial fluid leukocyte count and differential represents a simple, rapid, and accurate test for differentiating prosthetic joint-associated infection from aseptic failure. The cut-off values for diagnosing prosthetic joint infection are considerably lower than the one for septic arthritis in native joints. A synovial fluid leukocyte count greater than $1.7 \times 10^{3} / \mu \mathrm{L}$ and differential greater than $65 \%$ neutrophils had a sensitivity for diagnosing prosthetic joint infection of $94 \%$ and $97 \%$, and specificity of $88 \%$ and $98 \%$, respectively [6].

\section{Histopathology}

Histopathologic examination of the tissue surrounding the implant demonstrates a high sensitivity (> 80\%) and a high specificity (>90\%) [2]. Acute inflammation has been variably defined as from 1 to 10 or greater neutrophils per high-power field. Because the degree of infiltration with inflammatory cells may considerably vary between tissue sections, at least 10 high-power fields should be examined to obtain an average count. A major limitation of histopathology examination is the lack of identification of the causative microorganism, an essential element in selecting appropriate antimicrobial therapy. Furthermore, interpretation of tissue histopathology from patients with an 
underlying systemic inflammatory disorder (eg, rheumatoid arthritis or psoriasis) may be difficult.

\section{Microbiology}

Preoperative aspirate of fluid collections and intraoperative tissue cultures provide the most accurate specimens for detecting the infecting microorganism. At least three intraoperative tissue areas should be sampled for microbiology. Swabs are not adequate because of their low sensitivity. If possible, the clinician should discontinue any antimicrobial therapy at least 2 weeks before tissue sampling for culture.

Using sonication to dislodge microorganisms from the surface of explanted devices significantly increases the sensitivity of culture, especially when a patient is receiving antimicrobial treatment. When explanted implants were sonicated in polyethylene bags, the culture sensitivity of the sonication fluid was superior to that of standard periprosthetic tissue $(75 \%$ vs $54 \%)$, but specificity was insufficient due to bag leakage contaminating the sonication fluid [7]. Therefore, specimen sonication in bags should be avoided [8,9]. When sonications of 331 explanted hip and knee prostheses were studied in solid containers, the sensitivity of sonication fluid cultures was higher than those of periprosthetic tissue $(79 \%$ vs $61 \%$, $P<0.001)$ with specificities for both of $99 \%[10 \bullet \bullet]$. Implant sonication was especially helpful in patients who received prior antimicrobial therapy. In addition, sonicate cultures identified mixed infections more frequently than periprosthetic tissue cultures, indicating that at least some mixed infections were previously missed by conventional tissue cultures. In addition, more than one morphologic type of the same organism was frequently found in sonicate cultures, indicating that microorganisms exist in biofilms in different growth stages on a continuous scale. Interestingly, some morphologic types exhibit different antimicrobial susceptibility; however, the relevance of this needs to be determined. Quantitative molecular methods (eg, polymerase chain reaction) may further facilitate the diagnosis, especially in patients receiving antimicrobial treatment [11].

\section{Imaging studies}

Radionuclide studies depict physiologic processes preceding anatomic changes. Bone scintigraphy with ${ }^{99 \mathrm{~m}} \mathrm{Tc}$ alone is not useful to detect implant-associated infection due to its low specificity [12]. During the first postoperative year, increased bone remodeling is normally present around the prosthesis. In addition, aseptic loosening cannot be differentiated from infection. Therefore, bone ${ }^{99 m}$ Tc scintigraphy should be combined with ${ }^{99 m}$ Tc-ciprofloxacin (Infecton) scan, with ${ }^{99 \mathrm{~m}} \mathrm{Tc}$-hexamethylpropylene amine oxime (HMPAO) leukocyte scintigraphy, or with ${ }^{99 m}$ Tc-labeled antigranulocyte monoclonal antibodies. The values for sensitivity, specificity, and accuracy were $94 \%, 83 \%$, and $89 \%$ for Infecton, and $63 \%, 97 \%$, and
$77 \%$ for labeled leukocytes [13]. Similarly, in another study, scintigraphy with ${ }^{99 \mathrm{~m} T c-l a b e l e d ~ m o n o c l o n a l ~ a n t i-~}$ granulocyte antibodies had an accuracy of $81 \%$ to detect prosthetic joint infection [14]. However, this technique is not available in the United States.

The role of fluorine-18-fluorodeoxyglucose positron emission tomography for differentiation of infection and aseptic loosening in total hip arthroplasty is still controversial. Whereas Love et al. [15] reported a low specificity of $9 \%$ and a high sensitivity of $100 \%$, Stumpe et al. [16] found a high specificity of $83 \%$ and a low sensitivity of $28 \%$. Obviously, this technique needs further evaluation for implant imaging.

Conventional radiographs are of limited value in the workup of prosthetic joint-associated infection. Low specificity is the main limitation, because radiolucency, osteolysis, and migration are observed not only in patients with infection, but also in those with aseptic loosening [16]. By performing serial radiographs, prosthetic migration can be measured. Rapidly progressive periprosthetic osteolysis and/or irregular periprosthetic osteolysis are suggestive for infection [16].

Contrast arthrography is useful in the assessment of implant stability in patients with total hip arthroplasty [17]. During the procedure, synovial fluid can be sampled for differential cell counts and microbiologic analysis. Synovial outpouchings and abscesses are typical signs of infection. The resolution may be improved by using digital subtraction technique.

Ultrasonography can be used to detect synovial fluid and to guide joint aspiration and drainage procedures. It is especially helpful in prosthetic hip infection in which effusion cannot be clinically diagnosed.

Computed tomography (CT) facilitates the detection of joint effusion, sinus tracts, soft tissue abscesses, bone erosion, and periprosthetic lucency, although metallic artifacts reduce image quality. By using a special technique, such artifacts can be minimized, allowing detection of loosening and signs of soft tissue infection.

Magnetic resonance imaging (MRI) can be safely used in patients with nonferromagnetic implants. MRI displays greater resolution for soft tissue abnormalities than CT or radiography, and greater anatomic detail than radionuclide scans. Similar to CT, the main disadvantages of MRI are imaging interferences in the vicinity of metal implants.

\section{Management \\ General aspects}

The treatment goal is an infection-free, pain-free, functional joint. Thus, neither lifelong suppressive antimicrobial therapy nor prosthesis removal without replacement reaches this goal. In some centers, the standard treatment of prosthetic joint-associated infection still includes either a two-stage prosthesis exchange with meticulous removal of all foreign 


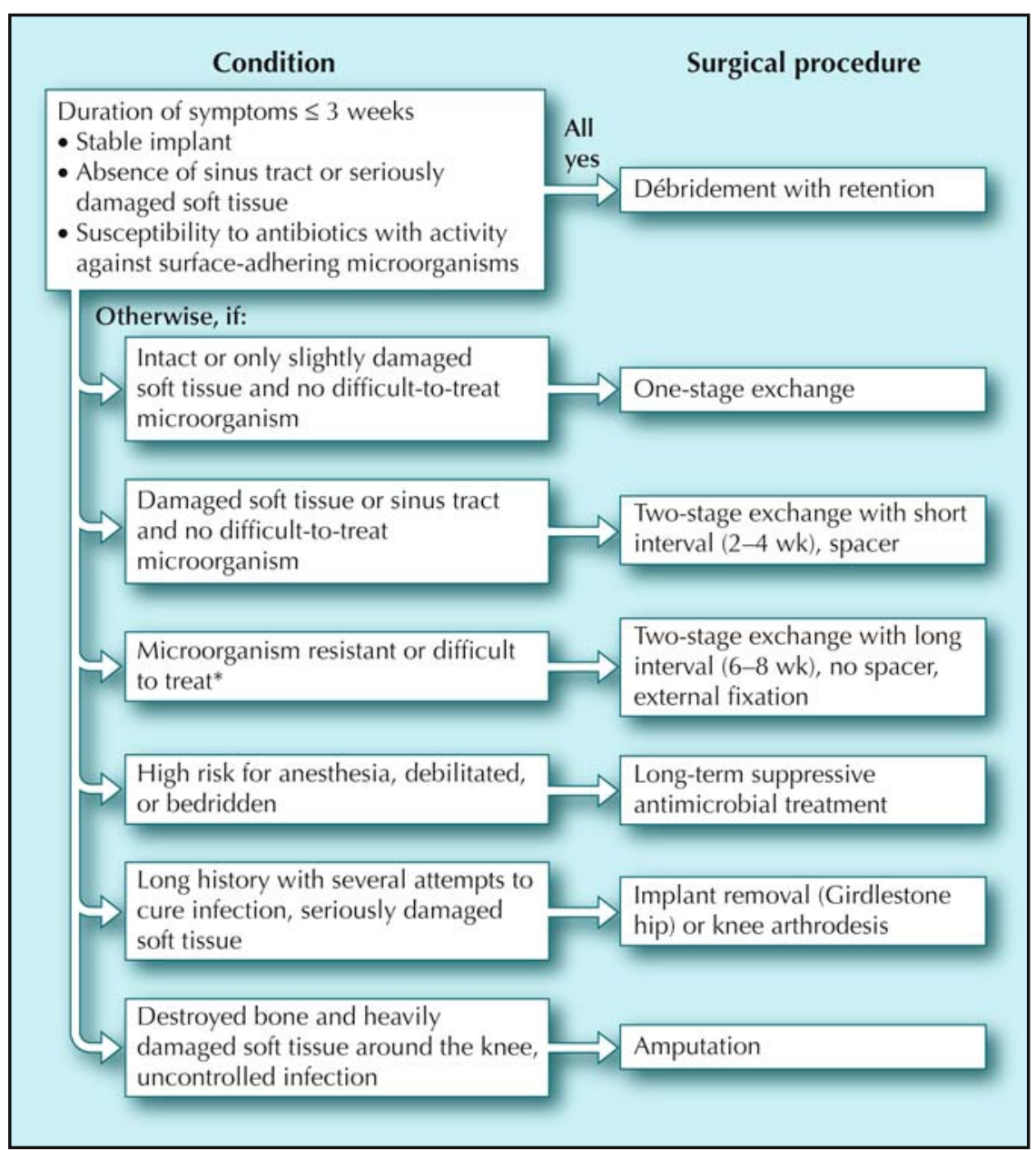

Figure 1. Surgical treatment algorithm for prosthetic joint-associated infections. Asterisk indicates difficult-to-treat microorganisms: methicillin-resistant Staphylococcus aureus, enterococci, small-colony variants of staphylococci, quinolone-resistant Pseudomonas aeruginosa, any type of multidrug-resistant microorganism, and fungi.

material combined with a 6-week course of intravenous antibiotics or retention of the prosthesis combined with long-term suppressive antibiotic treatment [18]. However, according to newer studies and concepts, this approach is no longer justified. The least invasive procedure resulting in cure-not merely suppression of infection-should be chosen, combined with long-term antimicrobial treatment [19-21]. The cornerstone of successful treatment is an early microbiologically confirmed diagnosis, because surgical treatment can be less invasive in patients with a short history of infection (see below). This can be achieved by a high degree of clinical suspicion and avoidance of empirical antibiotic therapy without unequivocal diagnosis.

\section{Surgical interventions}

Due to the heterogeneity of the cases, no controlled trials comparing different surgical options are available or will likely be available in the future. Therefore, surgical treatment is generally based on personal experience of orthopedic surgeons and differs considerably between individual centers. Nevertheless, current concepts are based on in vitro experiments, animal models, observational clinical studies, and a controlled trial [19-24]. The first question is whether the treatment goal is curative (elimination of microorganisms) or palliative (suppression of bacterial growth). Figure 1 shows the algorithm for the optimal strategy in each clinical situation; this algorithm has been validated in two cohort studies involving infections of total hip and knee prosthesis with an overall success rate greater than $80 \%$ for the first treatment attempt $[25,26 \bullet]$. In addition, an external validation has been published recently [27]. Antimicrobial treatment of implant-associated infection without any surgical intervention usually results in failure. The following surgical options should be chosen according to well-defined criteria [2].

\section{Débridement with retention}

The success rate of this procedure is widely underestimated, because patients were often included without proper selection and it has often been used for suppression, not cure [28-31]. The success rate is as high as for exchange surgery ( $>80 \%)$ if the following conditions are fulfilled: 1) a stable implant, 2) a pathogen with susceptibility to antimicrobial agents active against surface-adhering microorganisms, 3) absence of a sinus tract or a periprosthetic abscess, and 4) duration of infection symptoms less than 3 weeks $[22,24,25,26 \bullet]$.

\section{One-stage exchange}

One-stage exchange includes removal and reimplantation during the same surgical procedure. Patients with intact or 
only slightly compromised soft tissue qualify for this procedure, reaching a success rate of $86 \%$ to $100 \%[32,33]$. A two-stage revision with a long interval is preferred if resistant or difficult-to-treat microorganisms are causing the infection, such as methicillin-resistant $S$. aureus (MRSA), small-colony variants of staphylococci [34•], enterococci, quinolone-resistant Pseudomonas aeruginosa, or fungi.

Two-stage exchange

Two-stage exchange includes removal of the prosthesis with implantation of a new prosthesis during a later surgical procedure. A short interval until reimplantation (2-4 weeks) and a temporary antimicrobial-impregnated bone cement spacer may be used in most cases. However, if difficult-to-treat microorganisms are isolated, a longer interval ( 8 weeks) without a spacer is preferred. The twostage procedure can be used for every patient and has a success rate generally exceeding $90 \%[2,35]$. However, the two-stage exchange is difficult and time-consuming, and the functional result may be suboptimal due to repetitive surgery resulting in bone and muscle destruction [2]. Therefore, it should be chosen when less invasive surgical modalities are not appropriate.

\section{Permanent removal}

Permanent removal of the device is usually reserved for patients with a high risk of reinfection (eg, severe immunosuppression, active intravenous drug use) or a functional improvement after reimplantation is not expected. Alternatively, long-term antimicrobial suppression may be chosen if the patient is inoperable, bedridden, or debilitated. However, suppressive therapy only controls clinical symptoms and infection relapse occurs in most patients $(>80 \%)$ when antimicrobials are discontinued.

\section{Bone cement}

Use of antibiotic-loaded bone cement is controversial because no double-blinded randomized studies are available [36]. In a prospective multicenter study involving 1688 patients with primary implant fixation, the infection rate was $0.8 \%$ vs $1.9 \%$ without antibiotic-loaded cement [37]. However, due to lack of controlled trials, the clinical efficacy of antibiotic-loaded cement remains uncertain in primary implantation. In contrast, the use of antibiotic-loaded bone cement in patients with hip prosthesis-associated infection treated with one-stage exchange is less controversial. In a summary of 29 studies, the cure rate was significantly higher in patients treated with antibiotic-impregnated bone cement $(86 \%$ vs $59 \%)$ [35].

Surgical therapy in patients with fracture-fixation devices The two treatment goals in patients with fracture-fixation devices are consolidation of the bone fracture and prevention of chronic osteomyelitis. Unlike in patients with prosthetic joints, eradication of infection is not always required because the device can often be removed after consolidation. There are different types of infection, namely pin-track infection, osteomyelitis after plating, and osteomyelitis after intramedullary nailing. We recently reviewed the treatment principles of these infections [38•].

\section{Antimicrobial therapy}

Table 2 summarizes antimicrobial agents according to the pathogen and its antimicrobial susceptibility [2]. In the presence of an implanted device, the type and length of antimicrobial therapy are crucial. Due to persistence of microorganisms in the biofilm, infection commonly recurs with inappropriate antibiotic choice and/or duration. The necessary duration of treatment is based on case series and one controlled study $[2,22]$. We suggest treatment duration of 3 months $(6$ months for knee prostheses), administered intravenously for the first 2 weeks, and followed by oral therapy if a drug with good oral bioavailability is available.

The treatment course can be shortened to 6 weeks if all foreign material is removed and no spacer implanted during a two-stage surgery. In this situation, persistence of a device-associated biofilm can be avoided. After 6 weeks of treatment, device reimplantation should be delayed by an additional 2 weeks to obtain reliable tissue specimens for culture to document treatment success. After reimplantation, antimicrobial therapy is initially readministered and then discontinued if intraoperative cultures remain negative; otherwise treatment is continued for 3 months $(6$ months for knee prostheses).

In cases of rifampin-susceptible staphylococcal implant infections, rifampin-combination regimens should be used, because of the excellent activity of rifamycins on slow-growing and adherent staphylococci [22]. These properties of rifampin have been shown in vitro, in animal models, and in several clinical studies [19-23,25,26•,39•]. Rifampin must be combined with another drug to prevent emergence of resistance in staphylococci. Newer rifamycin derivatives (eg, ABI-0043) have proved to have efficacy comparable to rifampin in an animal model of foreignbody infection [39•]. Unfortunately, emerging resistance is a problem common to all known rifamycins. Recently, a hybrid antibiotic compound, CBR-2092 (Cumbre Pharmaceuticals, Dallas, TX), a fusion rifamycin-quinolone drug, has been developed. This represents a new and interesting class of investigational antibiotics that can be given as monotherapy [40].

Fluoroquinolones are the main drugs combined with rifampin in staphylococcal infections $[2,22,23]$. Their chief advantages are good bioavailability, activity, and safety. Classic fluoroquinolones (eg, ciprofloxacin or pefloxacin) have been replaced by newer ones (moxifloxacin, levofloxacin, and gatifloxacin), which have an improved in vitro activity against quinolone-susceptible staphylococci and favorable pharmacokinetics. In contrast to older quinolones, no controlled clinical trials of implant-associated infection with a sufficient follow-up period have been performed. Newer 


\begin{tabular}{|c|c|c|c|}
\hline Microorganism & Antimicrobial agent* & Dose & Route \\
\hline \multicolumn{4}{|l|}{ Staphylococcus spp. } \\
\hline \multirow[t]{5}{*}{ Methicillin-susceptible } & Rifampin plus & $450 \mathrm{mg}$ every $12 \mathrm{~h}$ & $\mathrm{PO} / \mathrm{IV}$ \\
\hline & $\begin{array}{l}\text { Nafcillin or (flu)cloxacillin }{ }^{+} \\
\text {for } 2 \text { wk, followed by }\end{array}$ & 2 g every $6 \mathrm{~h}$ & IV \\
\hline & Rifampin plus & $450 \mathrm{mg}$ every $12 \mathrm{~h}$ & $\mathrm{PO}$ \\
\hline & Levofloxacin or & $\begin{array}{l}750 \mathrm{mg} \text { every } 24 \mathrm{~h} \text { to } 500 \mathrm{mg} \\
\text { every } 12 \mathrm{~h}\end{array}$ & $\mathrm{PO}$ \\
\hline & Ciprofloxacin & $750 \mathrm{mg}$ every $12 \mathrm{~h}$ & $\mathrm{PO}$ \\
\hline \multirow[t]{11}{*}{ Methicillin-resistant } & Rifampin plus & $450 \mathrm{mg}$ every $12 \mathrm{~h}$ & $\mathrm{PO} / \mathrm{IV}$ \\
\hline & Vancomycin or & 1 g every $12 h^{\ddagger}$ & IV \\
\hline & $\begin{array}{l}\text { Daptomycin } \\
\text { for } 2 \mathrm{wk} \text {, followed by }\end{array}$ & $6 \mathrm{mg} / \mathrm{kg}$ every $24 \mathrm{~h}$ & IV \\
\hline & Rifampin plus & 450 mg every $12 \mathrm{~h}$ & $\mathrm{PO}$ \\
\hline & Levofloxacin or & $\begin{array}{l}750 \mathrm{mg} \text { every } 24 \mathrm{~h} \text { to } 500 \mathrm{mg} \\
\text { every } 12 \mathrm{~h}\end{array}$ & $\mathrm{PO}$ \\
\hline & Ciprofloxacin or & 750 mg every $12 \mathrm{~h}$ & $\mathrm{PO}$ \\
\hline & Teicoplanin ${ }^{\S}$ or & 400 mg every $24 \mathrm{~h}$ & $\mathrm{IV} / \mathrm{IM}$ \\
\hline & Fusidic acid or & $500 \mathrm{mg}$ every $8 \mathrm{~h}$ & $\mathrm{PO}$ \\
\hline & Cotrimoxazole or & 1 DS tablet every $8 \mathrm{~h}$ & $\mathrm{PO}$ \\
\hline & Minocycline or & $100 \mathrm{mg}$ every $12 \mathrm{~h}$ & $\mathrm{PO}$ \\
\hline & Clindamycin & 300 mg every $6 \mathrm{~h}$ & $\mathrm{PO}$ \\
\hline \multirow[t]{3}{*}{ Streptococcus spp. } & Penicillin $\mathrm{G}^{\dagger}$ or & 5 million $U$ every $6 \mathrm{~h}$ & IV \\
\hline & ceftriaxone & $\begin{array}{l}2 \text { g every } 24 \mathrm{~h} \text { for } 4 \mathrm{wk} \text {, } \\
\text { followed by }\end{array}$ & IV \\
\hline & Amoxicillin & 750-1000 mg every $8 \mathrm{~h}$ & $\mathrm{PO}$ \\
\hline \multirow{3}{*}{$\begin{array}{l}\text { Enterococcus spp. } \\
\text { (penicillin-susceptible) }\end{array}$} & Penicillin G or & 5 million $U$ every $6 \mathrm{~h}$ & IV \\
\hline & $\begin{array}{l}\text { Ampicillin or amoxicillin plus } \\
\text { aminoglycoside** } \\
\text { for } 2-4 \text { wk, followed by }\end{array}$ & 2 g every $4-6 h$ & IV \\
\hline & Amoxicillin & 750-1000 mg every $8 \mathrm{~h}$ & $\mathrm{PO}$ \\
\hline $\begin{array}{l}\text { Enterobacteriaceae } \\
\text { (quinolone-susceptible) }\end{array}$ & Ciprofloxacin & $750 \mathrm{mg}$ every $12 \mathrm{~h}$ & $\mathrm{PO}$ \\
\hline \multirow[t]{2}{*}{$\begin{array}{l}\text { Nonfermenters } \\
\text { (eg, Pseudomonas } \\
\text { aeruginosa) }\end{array}$} & $\begin{array}{l}\text { Cefepime or ceftazidime plus } \\
\text { aminoglycoside** } \\
\text { for } 2 \text { to } 4 \text { wk, followed by }\end{array}$ & $2 \mathrm{~g}$ every $8 \mathrm{~h}$ & IV \\
\hline & Ciprofloxacin & $750 \mathrm{mg}$ every $12 \mathrm{~h}$ & $\mathrm{PO}$ \\
\hline \multirow[t]{2}{*}{ Anaerobes $^{t+}$} & $\begin{array}{l}\text { Clindamycin } \\
\text { for } 2-4 \text { wk, followed by }\end{array}$ & $600 \mathrm{mg}$ every $6-8 \mathrm{~h}$ & IV \\
\hline & Clindamycin & 300 mg every $6 \mathrm{~h}$ & $\mathrm{PO}$ \\
\hline \multicolumn{4}{|c|}{ 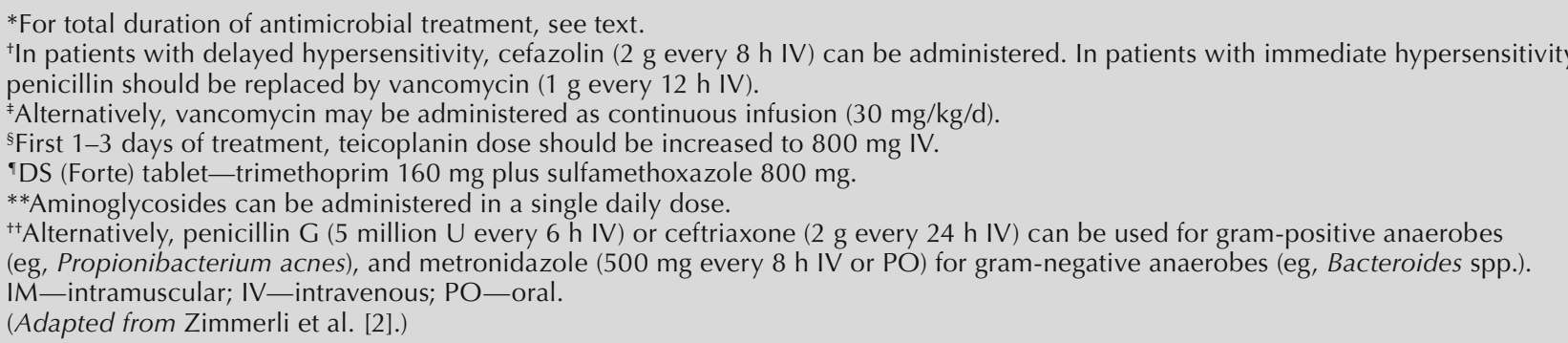 } \\
\hline
\end{tabular}




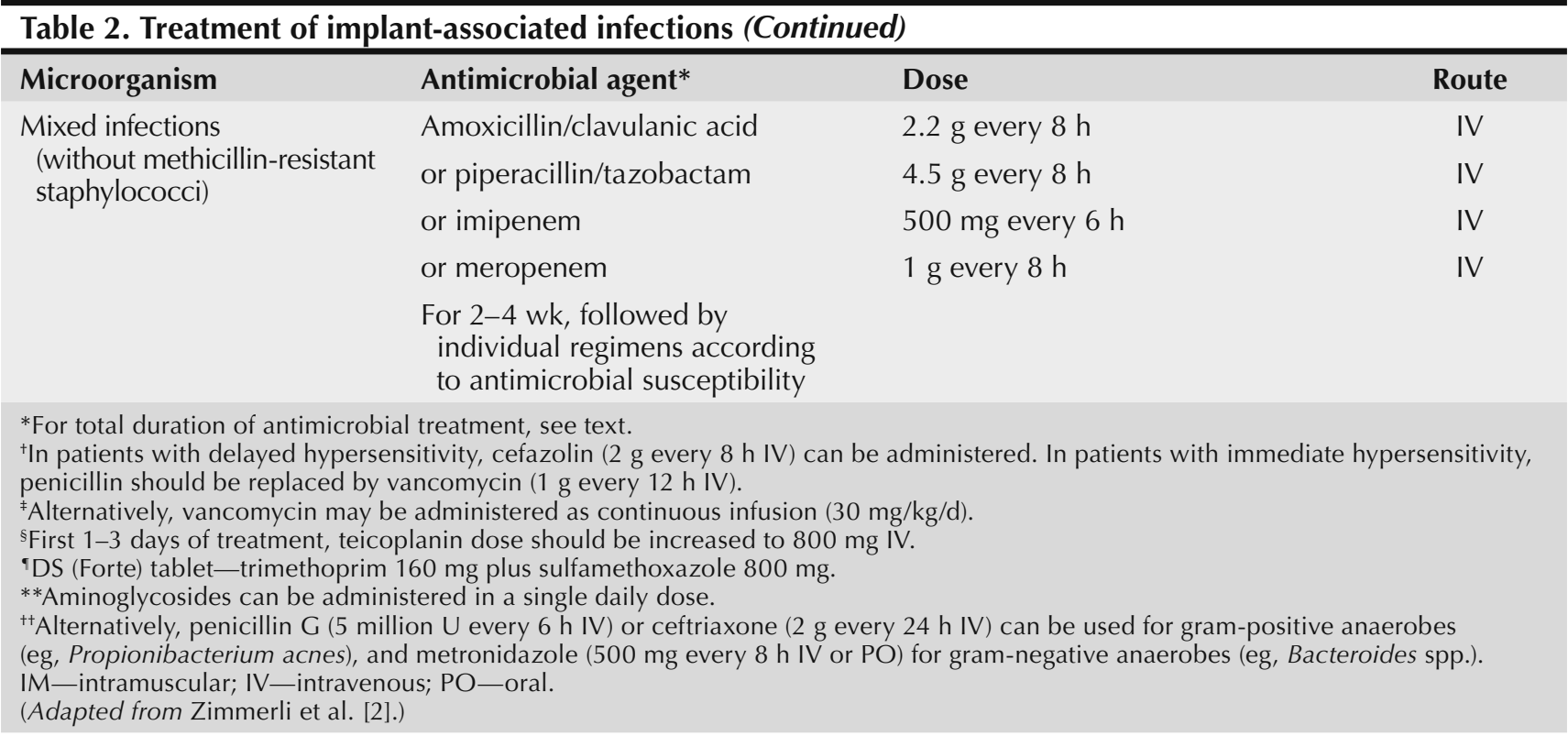

quinolones were studied in experimental bone infections [41], but only anecdotal clinical data exist for levofloxacin and moxifloxacin, respectively [26•]. One of the problems of long-term therapy with fluoroquinolones is the risk of tendonitis, which can occur with all fluoroquinolones, and is therefore a class effect.

Other oral combination partners for rifampin include cotrimoxazole, clindamycin, minocycline, and linezolid; however, clinical data in prosthetic joint infections are scarce. Nevertheless, such combinations have similar efficacy as quinolone combinations, because the crucial drug is rifampin and the partner drug is primarily serving to prevent the emergence of resistance. In contrast, more information is available on linezolid treatment $[42,43]$. In the study by Soriano et al. [42], 85 patients with acute or chronic orthopedic device-associated infections due to methicillin-resistant staphylococci were treated either with linezolid alone or with a linezolid/rifampin combination. The cure rate was high in both groups if the implant was removed $(92 \%$ and $100 \%$, respectively). In patients with retained implant and less than 2 weeks of infection symptoms, linezolid alone was less efficacious than in combination with rifampin $(60 \%$ vs $88 \%)$. However, in patients with chronic infection, the cure rate was low in both groups $(40 \%$ vs $47 \%)$. This confirms that débridement with retention should be reserved for patients with short duration of infection $[2,44]$. Toxicity is a major concern with prolonged linezolid treatment (> 21 days). Reversible myelosuppression is frequent $(10 \%)$ and irreversible neuropathy occasionally occurs [43].

Daptomycin is a lipopeptide antibiotic active against several gram-positive bacteria, including MRSA, vancomycin-resistant $S$. aureus, and vancomycin-resistant enterococci (VRE) [45]. Its efficacy has been tested in an animal model of implant-associated infections, where it showed no advantage compared with vancomycin or tei- coplanin [19]. In a recently published series of 12 patients with prosthetic joint-associated infection, a 6-week course with daptomycin $(4 \mathrm{mg} / \mathrm{kg}$ per day) failed in half of the cases [46]. In patients with more than 2 weeks of symptoms of infection, foreign material was removed and the failure rate was 2 of $7(26 \%)$. In contrast, the treatment failed in 4 of 5 patients $(80 \%)$ with débridement and implant retention. None of these patients received a combination with rifampin, which could explain the high failure rate of infections with implant retention.

\section{Hematogenous Infection}

Patients with orthopedic implants are at lifelong risk for hematogenous infection. Exogenous infections acquired either intra- or perioperatively are usually manifested during the first 2 years after implantation. During this same period, the risk of hematogenous infection is also higher and differentiating between exogenous and hematogenous infection is difficult, especially if the patient has no detectable distant focus. In two of our studies, we analyzed the pathogenesis of infection. We found that $43 \%(27 / 63)$ of the total hip arthroplasty-associated infections were hematogenously acquired. Three quarters of them were late infections [25]. In the second study, a series of total knee arthroplasty-associated infection, $37.5 \%$ were of hematogenous origin, and most of these were late infections [26•]. Similarly, in the study by Maderazo et al. [47] 35\% of the prosthetic joint-associated infections occurred after the first year. Thus, patients with orthopedic implants remain prone to implant-associated infection throughout their lives.

Studies on the pathogenesis of implant-associated infection show a locally acquired granulocyte defect $[48,49]$. Accordingly, the minimal infecting dose for an implantassociated abscess is as low as 100 colony-forming units 
(CFU) of $S$. aureus in an animal model and in human volunteers $[48,50]$. Thus, each implant is a "locus minoris resistentiae." Quantifying the risk for hematogenous infection is clinically important. At first glance, one would think that implants are endangered during episodes of bacteremia induced by manipulations such as dental work, especially teeth extraction. However, such episodes of bacteremia are of short duration and of low bacterial density. The number of CFU after dental extraction in children ranges between 1 and $28 \mathrm{CFU} / \mathrm{mL}$ blood and usually does not exceed 15 minutes [51,52]. We therefore tested the critical bacterial density in the bloodstream resulting in permanent infection associated with extravascular foreign bodies [53]. Intracardiac inoculation of $5 \times 10^{7}$ CFU S. aureus resulting in 103 $\mathrm{CFU} / \mathrm{mL}$ blood was required to selectively induce subcutaneous implant-associated infection [53]. Similarly, in a rabbit knee joint model, Blomgren et al. [54] found that several intravascular injections of high doses of $S$. aureus were required to get prosthetic knee infection. Thus, patients with orthopedic implants are endangered during episodes of prolonged high-density bacteremia, but clearly not by low-density bacteremia observed after different types of manipulations (eg, teeth extraction). After teeth extraction, one third of the microorganisms are viridans streptococci [52]. In a study of 189 episodes of late infection after total joint replacement, only $2.1 \%$ were due to viridans streptococci [55]. This clinically confirms that dental work does not seriously endanger orthopedic implants.

Taken together, hematogenous infections do not occur during occasional transient bacteremia, but rather during clinically relevant infections. Maderazo et al. [47] reported the highest risk during skin infection. Among 67 patients with late prosthetic joint-associated infection, $46 \%$ had primary skin infections as the origin, and $66 \%$ of them were caused by $S$. aureus. Among three patients in whom infection was considered of dental origin, one patient had a dental abscess and one had periodontitis. Only one occurred after dental extraction. In all three cases, the microorganism was not typical for dental origin (S. aureus, Staphylococcus epidermidis, and P. acnes). With regard to hematogenous seeding, $S$. aureus is associated with the highest risk for hematogenous prosthetic joint-associated infection. Murdoch et al. [56] reported a risk of $34 \%$ of hematogenous infection in 44 patients with a prosthetic joint and $S$. aureus bacteremia.

The 2003 Advisory Statement from the American Dental Association and the American Academy of Orthopaedic Surgeons on antibiotic prophylaxis for dental patients with prosthetic joints concludes that scientific evidence does not support that antibiotic prophylaxis is required to prevent hematogenous infection during dental treatment [57]. Nevertheless, prophylaxis should be considered in certain high-risk patients. However, this latter statement is not based on scientific data. We suggest performing necessary dental work before joint replacement and avoiding it during the first year after implantation, if possible. More important than antibiotic prophylaxis is the rapid and adequate treatment of any infection. Because skin infections such as cellulitis or erysipelas pose the greatest risk, the patient must be educated to consult an experienced physician immediately. In addition, during or after such infections, especially after $S$. aureus bacteremia, prosthetic joint-associated infection must be considered.

\section{Conclusions}

In the future, the apparent incidence of prosthetic jointassociated infection will increase further due to 1) better detection methods for microbial biofilms involved in prosthetic joint infections, 2) the growing number of implanted prostheses in the aging population, and 3) the increasing residency time of prostheses, which are at lifelong risk for hematogenous infection.

Better understanding of the interaction among microorganisms, implant, and host may further improve our current approach to the diagnosis and treatment of implant-associated infections. The controversy regarding suppressive versus curative therapy should be clarified. If the properly selected patient is treated with the correct procedure, in most cases cure can be reached, and no long-term suppressive antibiotic therapy is needed. The rational choice of the optimal treatment modality should be based on duration of the infection, stability of the implant, antimicrobial susceptibility of the pathogen, and condition of the surrounding soft tissue. If all criteria are correctly considered, cure rates between $70 \%$ and $90 \%$ are the rule.

\section{Disclosures}

The authors have received independent research grants from Pfizer, Cumbre Pharmaceuticals, and ActivBiotics.

\section{References and Recommended Reading}

Papers of particular interest, published recently, have been highlighted as:

- Of importance

- Of major importance

1. del Pozo JL, Patel R: The challenge of treating biofilmassociated bacterial infections. Clin Pharmacol Ther 2007, 82:204-209.

2. Zimmerli W, Trampuz A, Ochsner PE: Prosthetic-joint infections. N Engl J Med 2004, 351:1645-1654.

3. Steckelberg JM, Osmon DR: Prosthetic joint infection. In: Infections Associated with Indwelling Medical Devices, edn 3. Edited by Bisno AL, Waldvogel FA. Washington, DC: American Society for Microbiology; 2000:173-209.

4. Donlan RM: Biofilms: Microbial life on surfaces. Emerg Infect Dis 2002, 8:881-890.

5. Ochsner PE, Sirkin MS, Trampuz A: Acute infection. In: Principles of Fracture Management. Edited by Ruedi RP, Murphy WM. Stuttgart, Germany: Thieme Verlag; 2006. 
6. Trampuz A, Hanssen AD, Osmon DR, et al.: Synovial fluid leukocyte count and differential for the diagnosis of prosthetic knee infection. Am J Med 2004, 117:556-562.

7. Trampuz A, Piper KE, Hanssen AD, et al.: Sonication of explanted prosthetic components in bags for diagnosis of prosthetic joint infection is associated with risk of contamination. J Clin Microbiol 2006, 44:628-631.

8. Dora C, Altwegg M, Gerber C, et al.: Evaluation of conventional microbiological procedures and molecular genetic techniques for diagnosis of infections in patients with implanted orthopedic devices. J Clin Microbiol 2008, 46:824-825.

9. Esteban J, Gomez-Barrena E, Cordero J, et al.: Evaluation of quantitative analysis of cultures from sonicated retrieved orthopedic implants in diagnosis of orthopedic infection. J Clin Microbiol 2008, 46:488-492.

10.• Trampuz A, Piper KE, Jacobson MJ, et al.: Sonication of removed hip and knee prostheses for improved diagnosis of infection. N Engl J Med 2007, 357:654-663.

In this prospective study, sonication of explanted hip and knee prostheses to dislodge biofilms was investigated.

11. Trampuz A, Osmon DR, Hanssen AD, et al.: Molecular and antibiofilm approaches to prosthetic joint infection. Clin Orthop 2003, 414:69-88.

12. Smith SL, Wastie ML, Forster I: Radionuclide bone scintigraphy in the detection of significant complications after total knee joint replacement. Clin Radiol 2001, 56:221-224.

13. Sonmezoglu K, Sonmezoglu M, Halac M, et al.: Usefulness of $99 \mathrm{mTc}$-ciprofloxacin (Infecton) scan in diagnosis of chronic orthopedic infections: comparative study with 99mTc-HMPAO leukocyte scintigraphy. J Nucl Med 2001, 42:567-574.

14. Ivancevic V, Perka C, Hasart O, et al.: Imaging of lowgrade bone infection with a technetium- $99 \mathrm{~m}$ labelled monoclonal anti-NCA-90 Fab' fragment in patients with previous joint surgery. Eur J Nucl Med Mol Imaging 2002, 29:547-551.

15. Love C, Marwin SE, Tomas MB, et al.: Diagnosing infection in the failed joint replacement: a comparison of coincidence detection 18F-FDG and 111In-labeled leukocyte/99mTc-sulfur colloid marrow imaging. J Nucl Med 2004, 45:1864-1871.

16. Stumpe KD, Notzli HP, Zanetti M, et al.: FDG PET for differentiation of infection and aseptic loosening in total hip replacements: comparison with conventional radiography and three-phase bone scintigraphy. Radiology 2004 , 231:333-341.

17. Cheung A, Lachiewicz PF, Renner JB: The role of aspiration and contrast-enhanced arthrography in evaluating the uncemented hip arthroplasty. AJR Am J Roentgenol 1997, 168:1305-1309.

18. Brause BD: Infections with prostheses in bones and joints. In: Principles and Practice of Infectious Diseases, edn 6. Edited by Mandell GL, Bennett JE, Dolin R. New York: Elsevier, Churchill, Livingstone; 2005:1332-1337.

19. Widmer AF, Frei R, Rajacic Z, Zimmerli W: Correlation between in vivo and in vitro efficacy of antimicrobial agents against foreign body infections. J Infect Dis 1990, 162:96-102.

20. Widmer AF, Wiestner A, Frei R, Zimmerli W: Killing of nongrowing and adherent Escherichia coli determines drug efficacy in device-related infections. Antimicrob Agents Chemother 1991, 35:741-746.

21. Zimmerli W, Frei R, Widmer AF, Rajacic Z: Microbiological tests to predict treatment outcome in experimental device-related infections due to Staphylococcus aureus. J Antimicrob Chemother 1994, 33:959-967.

22. Zimmerli W, Widmer AF, Blatter M, et al.: Role of rifampin for treatment of orthopedic implant-related staphylococcal infections: a randomized controlled trial. Foreign-Body Infection (FBI) Study Group. JAMA 1998, 279:1537-1541.
23. Widmer AF, Gaechter A, Ochsner PE, Zimmerli W: Antimicrobial treatment of orthopedic implant-related infections with rifampin combinations. Clin Infect Dis 1992, 14:1251-1253.

24. Trebse R, Pisot V, Trampuz A: Treatment of infected retained implants. J Bone Joint Surg Br 2005, 87B:249-256.

25. Giulieri SG, Graber P, Ochsner PE, Zimmerli W: Management of infection associated with total hip arthroplasty according to a treatment algorithm. Infection 2004 , 32:222-228.

26. Laffer RR, Graber P, Ochsner PE, Zimmerli W: Outcome of prosthetic knee-associated infection: evaluation of 40 consecutive episodes at a single centre. Clin Microbiol Infect 2006, 12:433-439.

Cohort study in patients with prosthetic knee-associated infection, showing that the outcome of different surgical procedures (débridement with retention, direct exchange, two-stage exchange) is similar, as long as the patients are treated according to a rational algorithm.

27. Betsch BY, Eggli S, Siebenrock KA, et al.: Treatment of joint prosthesis infection in accordance with current recommendations improves outcome. Clin Infect Dis 2008, 46:1221-1226.

28. Brandt CM, Sistrunk WW, Duffy MC, et al.: Staphylococcus aureus prosthetic joint infection treated with debridement and prosthesis retention. Clin Infect Dis 1997, 24:914-919.

29. Tsukayama DT, Wicklund B, Gustilo RB: Suppressive antibiotic therapy in chronic prosthetic joint infections. Orthopedics 1991, 14:841-844.

30. Crockarell JR, Hanssen AD, Osmon DR, Morrey BF: Treatment of infection with debridement and retention of the components following hip arthroplasty. J Bone Joint Surg Am 1998, 80:1306-1313.

31. Marculescu CE, Berbari EF, Hanssen AD, et al.: Outcome of prosthetic joint infections treated with debridement and retention of components. Clin Infect Dis 2006, 42:471-478.

32. Ure KJ, Amstutz HC, Nasser S, Schmalzried TP: Directexchange arthroplasty for the treatment of infection after total hip replacement. An average ten-year follow-up. J Bone Joint Surg Am 1998, 80:961-968.

33. Raut VV, Siney PD, Wroblewski BM: One-stage revision of infected total hip replacements with discharging sinuses. J Bone Joint Surg Br 1994, 76:721-724.

34.• Sendi P, Rohrbach M, Graber P, et al.: Staphylococcus aureus small colony variants in prosthetic joint infection. Clin Infect Dis 2006, 43:961-967.

Case series of patients with small colony variants (SCV) S. aureus in prosthetic joint infection, showing the importance of active search for SCV in treatment failure.

35. Langlais F: Can we improve the results of revision arthroplasty for infected total hip replacement? J Bone Joint Surg Br 2003, 85:637-640.

36. van de BH, Neut D, Schenk W, et al.: Staphylococcus aureus biofilm formation on different gentamicin-loaded polymethylmethacrylate bone cements. Biomaterials 2001, 22:1607-1611.

37. Josefsson G, Gudmundsson G, Kolmert L, Wijkstrom S: Prophylaxis with systemic antibiotics versus gentamicin bone cement in total hip arthroplasty. A five-year survey of 1688 hips. Clin Orthop Relat Res 1990, 173-178.

38. Trampuz A, Zimmerli W: Diagnosis and treatment of infections associated with fracture-fixation devices. Injury 2006, 37:S59-S66.

A review of the diagnostic approach and treatment concepts of infections associated with internal osteosynthesis.

39. Trampuz A, Murphy CK, Rothstein DM, et al.: Efficacy of a novel rifamycin derivative, ABI-0043, against Staphylococcus aureus in an experimental model of foreign-body infection. Antimicrob Agents Chemother 2007, 51:2540-2545.

Evaluation of a new rifamycin derivative in the treatment of a staphylococcal implant-associated infection using an established guinea pig model. 
40. Robertson GT, Bonventre EJ, Doyle TB, et al.: In vitro evaluation of CBR-2092, a novel rifamycin-quinolone hybrid antibiotic: studies of the mode of action in Staphylococcus aureus. Antimicrob Agents Chemother 2008 (Epub ahead of print).

41. Shirtliff ME, Calhoun JH, Mader JT: Comparative evaluation of oral levofloxacin and parenteral nafcillin in the treatment of experimental methicillin-susceptible Staphylococcus aureus osteomyelitis in rabbits. J Antimicrob Chemother 2001, 48:253-258.

42. Soriano A, Gomez J, Gomez L, et al.: Efficacy and tolerability of prolonged linezolid therapy in the treatment of orthopedic implant infections. Eur J Clin Microbiol Infect Dis 2007, 26:353-356.

43. Bressler AM, Zimmer SM, Gilmore JL, Somani J: Peripheral neuropathy associated with prolonged use of linezolid. Lancet Infect Dis 2004, 4:528-531.

44. Zimmerli W, Ochsner PE: Management of infection associated with prosthetic joints. Infection 2003, 31:99-108.

45. Carpenter CF, Chambers HF: Daptomycin: another novel agent for treating infections due to drug-resistant grampositive pathogens. Clin Infect Dis 2004, 38:994-1000.

46. Rao N, Regalla DM: Uncertain efficacy of daptomycin for prosthetic joint infections: a prospective case series. Clin Orthop 2006, 451:34-37.

47. Maderazo EG, Judson S, Pasternak H: Late infections of total joint prostheses. A review and recommendations for prevention. Clin Orthop 1988, 299:131-142.

48. Zimmerli W, Waldvogel FA, Vaudaux P, Nydegger UE: Pathogenesis of foreign body infection: description and characteristics of an animal model. J Infect Dis 1982, 146:487-497.
49. Zimmerli W, Lew PD, Waldvogel FA: Pathogenesis of foreign body infection: evidence for a local granulocyte defect. J Clin Invest 1984, 73:1191-1200.

50. Elek SD, Conen PE: The virulence of Staphylococcus pyogenes for man; a study of the problem of wound infection. Br J Exp Pathol 1957, 38:573-586.

51. Lucas VS, Lytra V, Hassan T, et al.: Comparison of lysis filtration and an automated blood culture system (BACTEC) for detection, quantification, and identification of odontogenic bacteremia in children. J Clin Microbiol 2002, 40:3416-3420.

52. Coulter WA, Coffey A, Saunders ID, Emmerson AM: Bacteremia in children following dental extraction. J Dent Res 1990, 69:1691-1695.

53. Zimmerli W, Zak O, Vosbeck K: Experimental hematogenous infection of subcutaneously implanted foreign bodies. Scand J Infect Dis 1985, 17:303-310.

54. Blomgren G, Lundquist H, Nord CE, Lindgren U: Late anaerobic haematogenous infection of experimental total joint replacement. A study in the rabbit using Propionibacterium acnes. J Bone Joint Surg Br 1981, 63B:614-618.

55. Deacon JM, Pagliaro AJ, Zelicof SB, Horowitz HW: Prophylactic use of antibiotics for procedures after total joint replacement. J Bone Joint Surg Am 1996, 78:1755-1770.

56. Murdoch DR, Roberts SA, Fowler JV Jr, et al.: Infection of orthopedic prostheses after Staphylococcus aureus bacteremia. Clin Infect Dis 2001, 32:647-649.

57. American Academy of Orthopaedic Surgeons: Antibiotic prophylaxis for dental patients with total joint replacements; 2004. [Information statement 1014.] Available at http://www.aaos.org/about/papers/advistmt/1014.asp. Accessed June 2008. 